\title{
Preface
}

\section{The Journey from Fast Tracking to Enhanced Recovery}

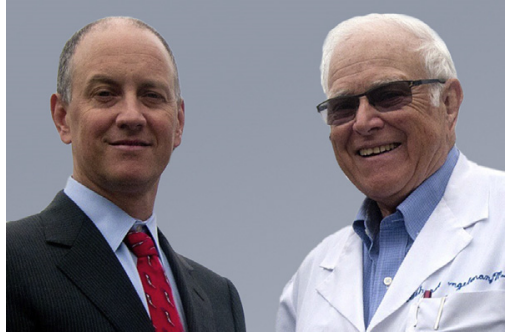

Daniel T. Engelman, MD, Editor, and Richard M. Engelman, MD

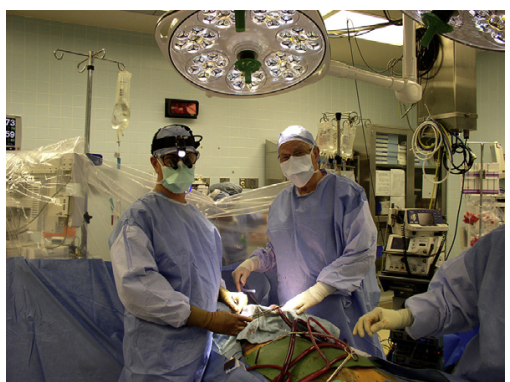

Daniel T. Engelman, MD and Richard M. Engelman, MD

My life's work in cardiac surgery began in 1968 at New York University, when I was a resident with Drs Frank Spencer and George Green. At that time, coronary bypass grafting (CABG) was just emerging as a therapeutic option for selected patients with symptomatic coronary artery disease. Dr Green had introduced internal mammary artery utilization, but saphenous veins were still our routine conduit of choice. Anesthesia was a combination of high-dose opioids, benzodiazepines, and long-acting inhalational agents. Patients remained intubated until at least the day after surgery and were on mandatory bedrest in the intensive care unit (ICU) for a minimum of 48 hours. Cardiac rehabilitation didn't begin before 72 hours and allowed only for very limited ambulation. Discharge was routinely 1 to 2 weeks after surgery, and patients were encouraged to remain sedentary for the next 4 to 6 weeks. These practices remained the standard for cardiac surgical perioperative care for decades, and morbidity following surgery was not inconsequential.

About 10 years later, I moved to Baystate Medical Center in Springfield, Massachusetts, where I founded the first cardiac surgical program in the western half of the state. In the early 1990s, I read an article by Bernard Krohn and colleagues in the Journal of Thoracic and Cardiovascular Surgery describing early discharge from The Hospital of the Good Samaritan in Los Angeles, and the excellent long-term results they achieved by paying close attention to perioperative care. ${ }^{1}$ With this in mind, in 1992 we began a collaboration with colleagues at Hartford Hospital in Connecticut to develop what we called a "Fast-Track" approach to care for CABG patients. Our process to care included the following: 
1. Recruitment of a Fast-Track Coordinator to oversee patient care

2. Development of an evidence-based Care Pathway

3. Documentation of compliance and an appreciation of Care Pathway variance with attention paid to deviation from the Pathway

4. Involvement of a multidisciplinary team, including anesthesiology and critical care

The results of our efforts were documented in a presentation at the American College of Surgeons Surgical Forum ${ }^{2}$ in 1993 and a 1994 publication in the Annals of Thoracic Surgery. ${ }^{3}$ A review of consecutive patients undergoing CABG surgery 6 months before $(N=282)$ and 6 months after $(N=280)$ program implementation found that $48 \%$ of Fast-Track patients could be discharged early (3 to 5 days after surgery), nearly double the rate prior to Fast-Track implementation, with no increases in morbidity or mortality. Extubation times, ICU stay, and overall hospital stay were also dramatically reduced. The critical components were early extubation ( $<8$ hours), limitation of intravenous fluids, control of atrial arrhythmias, normalization of gut function, and early ambulation. The discharge procedure incorporated the patient's family in the recovery process and utilized a Clinical Coordinator, Case Manager, Fast-Track Coordinator, and an outpatient Nurse Practitioner. As soon as it became apparent how effective this process was for CABG patients, we incorporated the same approach for all cardiac surgical procedures.

In 1993, the Baystate Cardiac Surgical program, together with Toronto General Hospital and Oxford Heart Centre, organized a live, international, multidisciplinary teleconference to describe and promote the Fast-Track approach, with attendees representing 40 cardiac surgical institutions. Since our first publication in 1994, there have been more than 700 peer-reviewed articles written about Fast-Track recovery in all surgical subspecialties. I am gratified that tens of thousands of patients have benefited from shorter lengths of stay and improved outcomes following all types of surgery, based on these guiding principles.

-Richard M. Engelman, MD

In 1999, I followed in my father's footsteps, becoming a board-certified cardiothoracic surgeon after graduating from Brigham and Women's Hospital in Boston, Massachusetts. As my career progressed, I noticed waning interest in the FastTrack protocols popularized by my father in the 1990s, coincident with increasing provider concern about patient-reported outcomes and the costs associated with care. ${ }^{4}$ Unfortunately, the potential risks of readmissions secondary to an early discharge, or poor HCAHPS (Hospital Consumer Assessment of Healthcare Providers and Systems) survey scores from patients with perceived excessive pain, ${ }^{5}$ threatened to outweigh the benefits of these Fast-Track protocols.

Changes in cardiac surgical training and practice patterns also played roles. As percutaneous interventional techniques improved, they replaced some of the open cardiac surgical volume and changed workflows. There was an increased emphasis on reducing any and all delays between patient evaluation and surgical procedures. Preoperative optimization was at odds with this new urgency.

Furthermore, national and international cardiac surgical conferences mainly focused on technique while devoting far less time and didactic material to evidence-based best practices in perioperative care. Cardiac surgical training programs were shortened, and the emphasis on preoperative and postoperative care was reduced. Finally, postoperative patient care has increasingly become the responsibility of critical care specialists, as surgeons spent a greater proportion of their time in the operating room performing longer and more complex procedures. 
In 2017, after reading multiple articles about the success of the Enhanced Recovery After Surgery (ERAS) Programs in most surgical specialties, I and a group of likeminded cardiac surgeons, decided to explore opportunities for introducing these same principles to cardiac surgery. The national and international interest was tremendous. A few early studies were simultaneously being conducted outlining enhanced recovery protocols demonstrating improved outcomes within our specialty. ${ }^{6}$ What was old was now new again.

That same year, we formed the nonprofit ERAS Cardiac Society with the mission to improve surgical care and recovery through research, education, audit, and implementation of evidence-based best practices. The society's membership was distributed evenly among anesthesiologists, intensivists, and cardiac surgeons specializing in perioperative care. After a formal collaboration with the international ERAS Society, ${ }^{7}$ our first guidelines were published in 2019 in JAMA Surgery. ${ }^{8}$ The international interest in this topic has been extraordinary. This report has been downloaded over 140,000 times and was the most viewed article, in the highest-impact surgical journal in 2019. ${ }^{9}$

In this issue of Critical Care Clinics, we summarize 9 major topics in Enhanced Recovery After Cardiac Surgery. The articles begin with preoperative optimization and follow the patient through the intraoperative and, finally, postoperative phases of recovery.

Advances in cardiac surgical operative techniques and myocardial protection have dramatically improved outcomes. An unfortunate and unintended consequence is that $80 \%$ of the preventable morbidity and mortality following cardiac surgery is now occurring outside of the operating room. ${ }^{10}$ Our hope is that a renewed emphasis on evidence-based best practices and standardized perioperative care will reduce overall morbidity and mortality and improve patient-centric care.

The Fast- Track recovery protocols that began under my father's leadership in 1992 will continue to evolve as more data are published from around the world. What was initially designed to speed up discharge has now evolved to emphasize improvement in each patient's perceptions of their ability to return to their presurgical state with the least discomfort, the fewest complications, and a minimal interruption in their daily lives.

This issue of Critical Care Clinics would never have been possible without the extraordinary contributions of the true "father" of Enhanced Recovery After Cardiac Surgery, Dr Richard Engelman. It is therefore dedicated to all of those patients who have already benefited and who will benefit in the future from special attention to standardizing evidence-based best practices in perioperative care. I would like to personally thank my father for starting us on this long but worthwhile journey.

-Daniel T. Engelman, MD

Daniel T. Engelman, MD

Richard M. Engelman, MD

Heart and Vascular Program

Baystate Health and University of Massachusetts Medical School-Baystate

759 Chestnut Street

Springfield, MA 01199, USA

E-mail address: 


\section{REFERENCES}

1. Krohn BG, Kay JH, Mendez MA, et al. Rapid sustained recovery after cardiac operations. J Thorac Cardiovasc Surg 1990;100(2):194-7.

2. Deaton DW, Engelman RM, Rousou J, et al. Fast track recovery of the cardiac surgical patient. Surg Forum 1993;44:223-5.

3. Engelman RM, Rousou JA, Flack JE 3rd, et al. Fast-track recovery of the coronary bypass patient. Ann Thorac Surg 1994;58(6):1742-6.

4. Shahian DM, Jacobs JP, Badhwar V, et al. Risk aversion and public reporting. part 1: observations from cardiac surgery and interventional cardiology. Ann Thorac Surg 2017;104(6):2093-101.

5. Available at: https://www.cms.gov/Medicare/Quality-Initiatives-Patient-Assess ment-Instruments/HospitalQualitylnits/HospitalHCAHPS. Accessed August 4, 2020.

6. Williams JB, McConnell G, Allender JE, et al. One-year results from the first USbased enhanced recovery after cardiac surgery (ERAS Cardiac) program. J Thorac Cardiovasc Surg 2019;157(5):1881-8.

7. Available at: https://www.businesswire.com/news/home/20180619006294/en/ ERAS-Cardiac-Surgery-Officially-Joins-ERAS-Society. Accessed August 4, 2020.

8. Engelman DT, Ben Ali W, Williams JB, et al. Guidelines for perioperative care in cardiac surgery: enhanced recovery after surgery society recommendations. JAMA Surg 2019;154(8):755-66.

9. Kibbe MR. JAMA Surgery-The Year in Review, 2019. JAMA Surgery 2020;155: 377-8.

10. Shannon FL, Fazzalari FL, Theurer PF, et al. A method to evaluate cardiac surgery mortality: phase of care mortality analysis. Ann Thorac Surg 2012;93(1):36-43. 